\title{
Analysis and Countermeasure of Plastic Film Mulching to Soil Pollution Quanke Zhao
}

College Tourism and Resource Environment, Zaozhuang University, Zaozhuang Shandong, 277160, China

Keywords: Plastic film mulching, Soil, Pollution.

\begin{abstract}
In recent years, plastic film mulching in our country has been widely applied to agriculture and well promote the development of agricultural industry. However, the residual pollution of plastic film mulching affects the better development of agriculture, because when the age limit of plastic film mulching is constantly prolonged, mulching film would be residual in soil. And the decomposing speed of mulching film is very slow and the recovery is very low, thus the plastic film residue in soil gradually accumulated, resulting in soil pollution and crop yield declining. This article analyzes the pollution status of plastic film mulching in our country, discusses soil pollution hazard and its reason and proposes several countermeasures.
\end{abstract}

\section{Introduction}

With the rapid development of the agricultural modernization, the applied mulching film has become an important method to guarantee agricultural high yield and stable yield. The plastic film mulching can be regarded as a very mature modern agricultural technology, which could not only achieve water retention and fertilizer maintenance, guarantee humidity and promote ground temperature but also well promote and prolong growing period of agricultural products to increase crop yield. However, mulching film at the same time is a high-molecular compound and hard to degrade. It would not be eroded by microorganism and degrade by itself, whose degradation period is usually 200 years to 300 years, with many toxic substances during degradation. When the age limit of plastic film mulching is continually prolonged, once the residual mulching film can not be rationally recycled, plastic film residue in soil would be gradually increased which would result in damage to soil structure, go against assimilation and growth of crop root system to water, fertilizer, etc. Thus the underground water can not continue infiltration and the soil would generate secondary salinization which would further affect the reduction of soil and crop yield. As time goes by, it would result in soil pollution hazard that later generations can not solve and bring huge threat to the development of agriculture.

\section{Pollution Status of Plastic Film Mulching in Our Country}

Firstly, the current total biodegradation of mulching film is relatively low. Biodegradation refers to the decomposition phenomenon generated by some high quality substances making use of live biological actions. The most common live creature is the microorganism, such as bacteria which would finally convert the compound into carbon dioxide and water. Degradation of different substances would be different. Degradation of some substances would be very fast and safe. And some may become smaller molecules during degradation which belong to primary degradation. The molecules are just the middle-type product by biodegradation. What is different from such elements as environment is that they would keep longer pollution and more toxicity.

Secondly, the continually increase of usage amount and areas of plastic film mulching result in more and more amount of residue and thus the pollution is more and more severe. The plastic film mulching was once regarded as the third revolution in agricultural field. However, none other than its 
function causes the improvement of usage amount and cover areas. According to a survey, the number of mulching film is gradually increased in the past ten years in our country. The mulching film residue in soil is usually distributed in plough layer, approximately concentrating on soil of 0 to 10 centimeters thick and reaching about $2 / 3$ of the mulching film residue. And the rest mulching film residue mainly distribute in soil of 10 to 30 centimeters thick. There would be little in soil more than 40 centimeters thick. The mulching film residue, the size and the shape in plough layer are all different. Influenced by agricultural activity and use pattern of mulching film, the mulching film residues are always flake, globular or coiling block, horizontally, aslant or vertically distributing in the soil. The fragment areas are different as well, appearing several sizes.

Thirdly, the policy of agricultural plastic mulching film is not in place. Famers belong to vulnerable groups, without enough knowledge reserve, fund or status. Making the application and cycling of mulching film residue perfect can not be realized in reality. However, when it comes to the disposition stimulation of mulching film, relevant policies are rather few. Thus only to provide a favorable environment for farmers and introduce reasonable policies to increase their economic income and prevent mulching film residue could better reduce soil pollution caused by plastic film mulching.

\section{Soil Pollution Hazard and Causes of Plastic Film Mulching Residue}

The mulching film residue in the soil would affect the soil physical property and greatly restrain the growth and development of each crop. The most important element of agricultural plastic mulching film is the high-molecular compound. Under natural condition, the above high polymeric compounds can not be decomposed. Once they are left in the soil for a long time, it would greatly influence the air permeability, water and fertilizer operation in soil. Since the plastic film residue in soil would change or excise the continuity of interior interspaces in soil, it would cause rather big pressure when the gravitational water move. When going down, gravitational water would move rather slowly and the infiltration capacity of water is reduced with the increase of mulching film. The reduction of water content in soil would lower drought resistance of plough, and thus the underground water would be hard to infiltrate down, with worse results such as soil salinization, etc. Plastic film residue causes the nonuniform watering and nutrient allocation, further lead to the decline of soil ventilation, influence normal soil structure and finally result in the decline of soil fertility.

The reasons why mulching film pollution is generated could mainly reduced to four points. Firstly, the current quality standard of agricultural film in our country is not high. Due to unduly pursue high economic benefit, current agricultural film thickness with the largest usage amount is usually 0.005 millimeters to 0.008 millimeters, which are very fragile to break so that cleaning and recycling work would be very difficult. Secondly, labor cost to recycle and process the waste mulching film is rather high and the reuse ratio very low. Thirdly, recycling the agricultural film mainly adopts labor gathering with high labor intensity, labor consumption and time consumption, resulting rather low subjective initiative of farmers to recycle mulching film. Fourthly, it has not established management mechanism of recycling and application of waste mulching film or such policies to implement recycle of plastic film residue. Thus enterprises are not willing to participate in recycle.

\section{Several Countermeasures to Ease Soil Pollution by Plastic Film Mulching}

Firstly, it should pay attention to environmental protection publicity and promote environmental awareness of farmers. Governments at all levels, especially relevant departments such as agriculture, environmental protection, etc. should constantly intensify publicity and education, promote farmers' initiative to participate in renovation of mulching film pollution to protect environment as well as their subjective initiative to recycle mulching film. They should rationally make use of multiple media publicities such as network, newspapers and periodicals, television, etc. to promote farmers' scientific understanding that mulching film residue would pollute soil, affect agricultural 
productivity and harm human health, correlate the soil preparation of farmers and recycling mulching film and reduce pollution level of agricultural plastic mulching film to environment.

Secondly, it should introduce mechanization recycle technology of the waste mulching film. It finds that most commercial crops planted in some western developed countries usually adopt plastic film mulching, with rather short period. It's worth noting that mulching films used in western countries are usually thick, about 0.015 millimeters. But thickness of mulching films used in our country is thinner, usually 0.006 millimeters to 0.008 millimeters. It asks us to strictly specify the production and application of the ultrathin mulching film, fully popularize and utilize mulching film with thickness more than 0.008 millimeter. If the thickness of mulching film is not enough, its intensity is relatively low and covering time is more longer than that in western countries. In this way, it causes such condition that mulching films used in our countries are easily broken and thus brings some difficulties in recycling. The traditional rolling mulching film recycling can not solve this problem. And introducing mechanization recycle technology would well solve this problem, whose recycling ways include such recycle technologies as tooth, drum-type, gear type, etc.

Thirdly, it should positively generalize new technology of optimum-period film uncovering. New technology of optimum-period film uncovering mainly means to uncover film before harvest rather than after harvest to select the most suitable film uncovering time of crops. There are five advantages of rational film uncovering. Firstly, it could shorten the film covering time to 60 to 90 days with rather good tenacity of mulching film. Thus it is easy for recycling and recycling rate should exceed 95\%, which could basically eliminate pollutions caused by plastic film residue in soil and protect the soil environment. Secondly, new technology of rational film uncovering could control and reduce soil humidity to prevent plant diseases and insect pests. Thirdly, new technology of rational film uncovering could promote development of crops' roots and air permeability of soil. Fourthly, rational film uncovering is usually implemented during the growth of crops when crops have plenty of moisture, at which time film uncovering could help roots better assimilate moisture and help the growth of crops. Fifthly, rational film uncovering could promote final-period management of crops, convenient for top application of crops in the middle and later periods. That is to say, rational film uncovering technology could not only promote recovery rate of mulching film but also save recycling cost to promote crop yield.

Fourthly, it should energetically popularize the application of degradable mulching film which mainly includes three kinds such as light degradation, biodegradation and light- biodegradation. Mulching film of light degradation is to add such photosensitizer as iron, salt, etc. to corresponding polymers as LLDPE, etc. By illumination, it could usually be degraded in about 90 days and even keep degradation to microorganism. However, light degradation is easily affected by factors such as geography, climate, etc. and thus could hardly be entirely degraded, which ask us to keep improving technical capacity of light degradation. Those countries such as Japan, Germany, etc. are devoted to use natural organism to degrade plastic film mulching substances. For example, adding soybeans or corn starch to polyethylene, with a few metallic compound, would produce catalytic action. Lightbiodegradation is to add starch or biological degradation agent to mulching film, whose plastic film residue could further militate with natural organism and change into low molecular compound, water and carbon dioxide, after light degradation.

Fifthly, it needs to make more strict disposal policy of plastic film mulching, with serious supervision. In order to lower soil pollution caused by mulching film, it must specify the production standard of mulching film, promote its manufacturing technique, pay high attention from the source of production and rationally make relevant norms in such aspects as production, use, sale, etc. of agricultural plastic mulching film. It should intensify work of technical research and development of degradable and non-pollution mulching film, rationally adjust specific production standard in the current manufacturing enterprises of agricultural plastic mulching film to guarantee such products with rather high quality, its thickness and tensile strength meeting national standard. On this basis, manufacturers with low-quality mulching films should be strictly investigated and punished. All farmers using ultra-thin mulching film that can not be recycled should pay some fees for pollution 
management. In this way, the source of mulching film pollution could be listed into the pathway of legalized management. At present, there are three levels or the general standard of agricultural plastic mulching film in China. Standard of the first level is that there are few mulching film residue in farmland which has no negative influence to the ecological environment of farmland and the growth of crops. Standard of the second level is that there are some mulching film residues in farmland which has no adverse influence to the growth and yield of crops. However, if the some mulching film residues exceed standard, crop failure would probably occur. Standard of the third level is that there are a lot of mulching film residues which would cause soil pollution and large-scale crop failure.

\section{Conclusion}

To sum up, soil is the general name of rather loose surface layer on the surface of land that plants could grow, as well as the important substance that all vital movements including mankind need. From the aspect of ecology, soil can be regarded as the main carrier that substance decomposer lives on and the outstanding content to realize circulation of materials. Using agricultural plastic film mulching technology could realize such purposes as water retention, high yield, etc. However, the plastic film residue in soil would easily result in soil pollution and affect the physical property, leading to lowered fertility of soil and slower plant growth speed with reduction of output. More severe situation is that long-term accumulation of plastic film residue would fully destroy production capacity of soil. Thus, fully governing soil pollution and protecting ecological environment of our farmland is the important step for the sustainable development of plastic film mulching. Thus, it needs to continually enhance publicity and education, promote the environmental consciousness of leaders and farmers to guarantee agriculture to get better development.

\section{References}

[1] Liang Zhihong, Wang Yong, Research Summary of Harm, Prevention and Cure of Farmland Mulching Film Residue in Our Country, China Cotton, 2012 (1).

[2] Wang Chunlan, On the Prevention and Cure of Plastic Film Mulching to Environmental Pollution, Agriculture \& Technology, 2013 (7).

[3] Yan Changrong, Liu Enke, Shu Fan, Characteristic of Plastic Film Mulching and Residual Pollution and Prevention and Control Technology in Our Country, journal of Agricultural Resources and Environment, 2014 (2).

[4] Zhou Lin, Influence Study of Economic Development Degree to Pollution Treatment of Agricultural Plastic Mulching Film, Anhui Agricultural Sciences, 2014 (19).

[5] Bai Yunlong, Pollution Status of Mulching Film Residue and Countermeasure Study of Plastic Film Residue Recycling in Inner Mongolia, Soil and Fertilizer Sciences in China. 2015 (6).

[6] Ren Wenjiang, Investigation of Farmland Mulching Film Usage and Residual Pollution in Huining County, Gansu Agricultural Science, 2016 (1). 